\title{
The familial Mediterranean fever (FMF) 50 score: does it work in a controlled clinical trial? Re-analysis of the trial of rilonacept for patients with colchicine resistant or intolerant FMF
}

\author{
P Hashkes $^{1 *}$, B Huang $^{2}$
}

From 8th International Congress of Familial Mediterranean Fever and Systemic Autoinflammatory Diseases

Dresden, Germany. 30 September - 3 October 2015

\section{Background}

The familial Mediterranean fever 50 score (FMF50) was recently devised to define response to treatment and as an outcome measure for clinical trials of FMF.

\section{Objectives}

To examine the performance of the FMF50 score in a previously published trial of rilonacept [1] for patients whose FMF was resistant or intolerant to colchicine.

\section{Methods}

We reanalyzed the data from the controlled trial of rilonacept vs. placebo in 14 patients with colchicine-resistant or intolerant FMF using the FMF50 score as the primary outcome. The FMF50 score required improvement by $\geq 50 \%$ in five of six criteria (attack frequency, attack duration, global patient assessment, global physician assessment, frequency of attacks with arthritis, and levels of acute-phase reactants) without worsening of the sixth criterion.

\section{Results}

In the original trial rilonacept was considered effective according to the primary outcome measure (differences in the attack frequency) with eight analyzable patients considered responders and four as non-responders. According to the FMF50 score, only two participants would have been considered responders to rilonacept, and one to placebo. Only two participants had $\geq 50 \%$ differences between rilonacept and placebo in five criteria.
The major explanation for non-response to treatment was that with rilonacept the duration of attack decreased by $\geq 50 \%$ in only 2 participants and 5 participants had no attacks of arthritis either during screening (before randomization) or during treatment with rilonacept.

\section{Conclusions}

The proposed FMF50 score did not differentiate well between responders and non-responders compared to the a priori defined primary outcome measure in this successful controlled study and should be revisited prior to adoption as a primary outcome measure in multinational FMF trials.

\section{Authors' details}

${ }^{1}$ Shaare Zedek Medical Center, Jerusalem, Israel. ${ }^{2}$ Cincinnati Children's Hospital Medical Center, Cincinnati, OH, USA.

\section{Published: 28 September 2015}

\section{Reference}

1. Hashkes PJ, Spalding SJ, Giannini EH, Huang B, Johnson A, Park G: Rilonacept for colchicine-resistant or -intolerant familial Mediterranean fever: a randomized trial. Ann Intern Med 2012, 157(8):533-541.

doi:10.1186/1546-0096-13-S1-P158

Cite this article as: Hashkes and Huang: The familial Mediterranean fever (FMF) 50 score: does it work in a controlled clinical trial? Re-analysis of the trial of rilonacept for patients with colchicine resistant or intolerant FMF. Pediatric Rheumatology 2015 13(Suppl 1):P158.

${ }^{1}$ Shaare Zedek Medical Center, Jerusalem, Israel

Full list of author information is available at the end of the article 